Signal \& Image Processing : An International Journal (SIPIJ) Vol.3, No.6, December 2012

\title{
JOINT AZIMUTH AND ELEVATION ANGLE ESTIMATION USING INCOMPLETE DATA GENERATED BY A FACULTY ANTENNA ARRAY
}

\author{
Yerriswamy T. ${ }^{1}$ and S.N. Jagadeesha ${ }^{2}$ \\ ${ }^{1}$ Proudhadevaraya Institute of Technology, T.B. Dam, Hospet, India \\ swamy_ty@yahoo.com \\ ${ }^{2}$ Jawaharlal Nehru National College of Engineering, Shivamogga, India \\ jagadeesha_2003@yahoo.co.in
}

\begin{abstract}
In this study, we extend the "Fault Tolerant Matrix Pencil Method for Direction of Arrival Estimation (DOA)" proposed by the authors [1] to joint estimation of Azimuth and Elevation Angles from a data generated by Uniform Planar Antenna array (UPA), where at random locations a few of the elements due to failure are missing. Joint Azimuth and Elevation Angles estimation is generally known as Twodimensional DOA estimation. In the proposed technique, the observed incomplete data is imputed first using the Matrix Completion (MC) algorithm and later the $2 D$ angles are jointly estimated using the Two dimensional Matrix Pencil Method (2D-MP). The resulting algorithm is robust in terms of failure of elements, is computationally efficient as it does not forms a correlation matrix and the angles are estimated using only a single snapshot. It is shown that the algorithm is able to estimate the DOAs when we have a fraction of the observed data. The numerical simulation results are provided to see the performance of the method for various incomplete data sizes and Signal-to-Noise Ratio (SNR).
\end{abstract}

\section{INTRODUCTION}

Joint estimation of azimuth and elevation angles generally known as Two Dimensional Direction of Arrival (2D-DOA) estimation is a key problem in array signal processing fields such as; radar, sonar, and wireless radio systems. There are a number of super resolution 2D DOA estimation methods like, Multiple Signal Classification (MUSIC) [2], Estimation of Signal Parameters via Rotational Invariance Technique (ESPRIT) [3]-[4]. These methods are subspace based approaches and require formation of correlation matrix and therefore a large number of noncoherent signal snapshots are required to effectively estimate the DOAs. On other hand, Direct Data Domain (D3) based Matrix Pencil (MP) method [5] [6] overcomes the requirement of correlation matrix, which makes it practically important.

Most of the DOA estimation techniques are devised for uniformly spatially sampled observed complete data sequences. However, estimating the 2D-DOAs with missing data due to failure of few elements is also important. Because, it is not possible to replace the faulty elements in all the situations. Estimating the DOAs from a faulty array is dealt in [7] - [8]. In [7] Larson and Stocia 
Signal \& Image Processing : An International Journal (SIPIJ) Vol.3, No.6, December 2012

proposed a ML estimate of the correlation matrix in the presence of the sensor failures and are shown to improve the performance of the MUSIC algorithm. Array failure correction based on Genetic algorithm is proposed by Yeo and Lu [9], which synthesises the original antenna array. A method for detection of the sensor failure and compensation has been proposed by Pirinen et al [10]. Vigneshwaran et al [8], use minimal resource allocation network to handle the sensor failures. These methods suffer from computational complexity, initialization, training, selection of network size etc.

In this paper, we extended the one dimensional fault tolerant MP method for DOA estimation published by authors in [1] to 2D DOA estimation. We use Matrix Completion via Convex Optimization proposed by Candes and Recht [11], to impute the missing 2D data and apply the 2D MP method proposed by Hua [5] to estimate the azimuth and the elevation angles. The proposed method results in a very low computationally complex algorithm and uses a single snapshot of the array. These features are important in many applications. In the matrix completion problem, if the given matrix is of rank ' $r$, and is not too structured, a small random subset of its entries allow to reconstruct it, exactly by solving a simple convex optimization program. Convex Optimization has been used in signal processing from a long time, a detailed discussion and applications on convex optimization can be found in [12] [13]. The problem of matrix completion is found in collaborative filtering [14], system identification [15] and computer networks[16]. Compared to the EM algorithm, MC algorithm is less complex and is also shown to be accurate in estimating the missing data. The problem of Matrix Completion is similar to Compressed Sensing (CS) [17] [18]. While MC recovers a rank deficient matrix, CS solves a system of underdetermined equations where the solution is sparse, i.e. have only a few non-zeros. The CS is also used DOA estimation in [19][20][21] in order to reduce the hardware complexity and the software requirements.

The rest of the paper is organized as follows. In section 2, the signal model for the 2D case is presented. In section 3, the matrix completion procedure is discussed. The conventional 2D MP method is revisited in section 4. The proposed MCMP method is discussed in section 5. The computer simulations are provided in section 6 , followed by the conclusion.

\section{SIGNAL MODEL}

Consider a Uniform Planar Array (UPA) of antenna of size My $\times \mathrm{Mz}$ elements with interelement spacing of $d$ equal to half of the wavelength of the signal source impinging on the array. An example of a UPA is shown in Fig. 1. It is assumed that $\mathrm{N}$ number of signals impinging on the array, such that the $\mathrm{i}^{\text {th }}$ source has an elevation angle of $\theta_{\mathrm{i}}$ and azimuth angle $\varphi_{\mathrm{i}}$. The noiseless data $\mathrm{x}(\mathrm{m}, \mathrm{n})$ measured at the feeding points of the omni directional antennas is given by

$$
x(m ; n)=\sum_{i=1}^{N} s_{i} e^{j \varphi_{i}} e^{j \frac{2 \pi}{\lambda w} d_{x} \sin \theta_{i} \cos \phi_{i}(m-1)} e^{j \frac{2 \pi}{\lambda w} d_{y} \sin \theta_{i} \sin \phi_{i}(n-1)}
$$

Where, $1 \leq \mathrm{m} \leq \mathrm{My}$ and $1 \leq \mathrm{n} \leq \mathrm{Mz}$. Equation 1 implies that $\mathrm{x}(\mathrm{m} ; \mathrm{n})$ consists of $\mathrm{N} 2$ 2-D sinusoids arriving at the array from elevation angle $\theta i$ and azimuth angle $\phi$ i. Further $s_{i}$ and $\varphi i$ are amplitude and phase respectively of the $i^{\text {th }}$ source, where $i=1,2, \ldots . N$. In the noisy case, the received data is 
Signal \& Image Processing : An International Journal (SIPIJ) Vol.3, No.6, December 2012

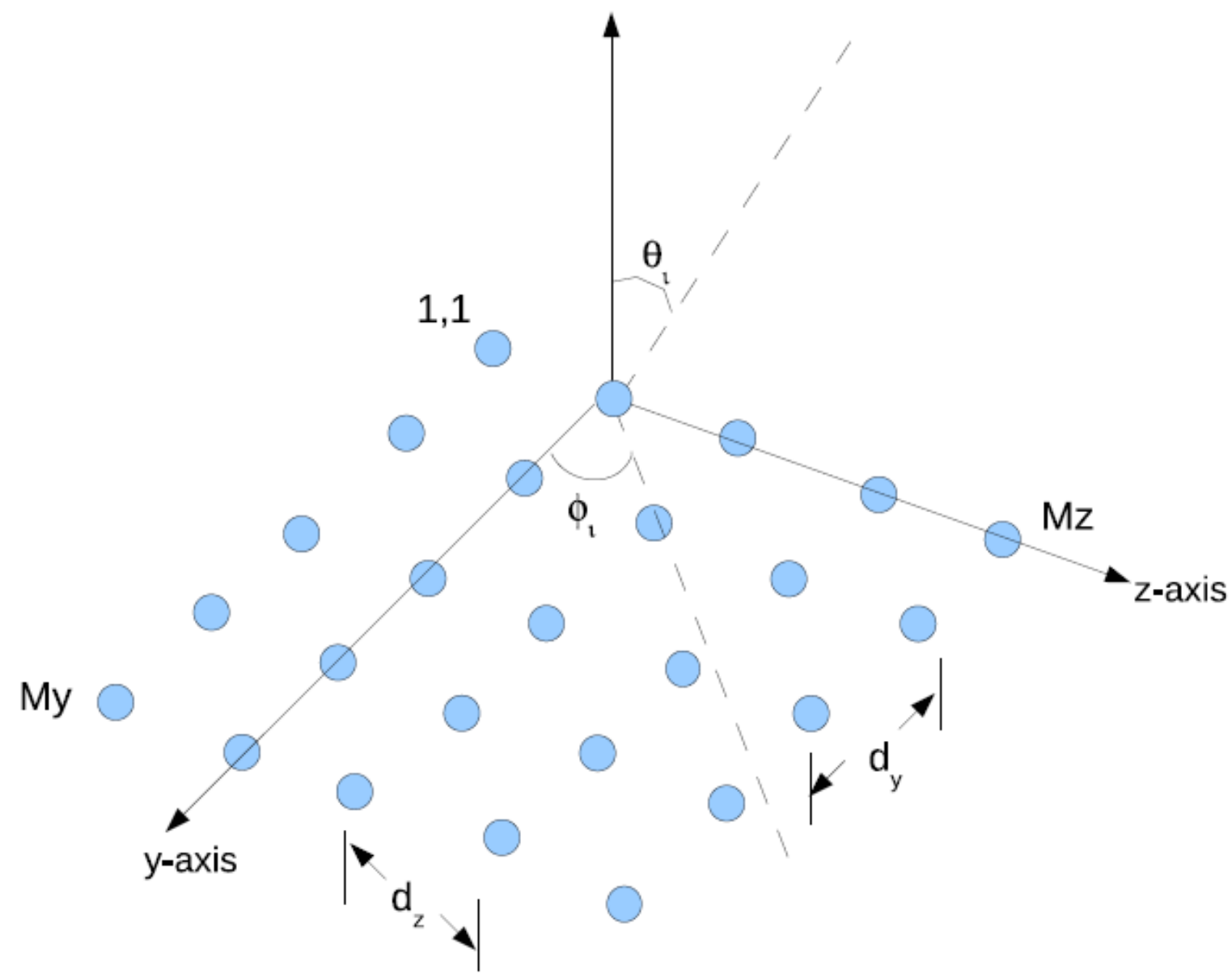

Figure 1: Uniform Planar Antenna Array

$$
x^{\prime}(m ; n)=x(m ; n)+w(m, n)
$$

Where $w(m ; n)$ is the 2-D noise sequence, and is assumed to be additive white Gaussian noise.

Equation 1 can be simplified to

$$
x(m ; n)=\sum_{i=1}^{N} \alpha_{i} z_{i}^{m-1} y_{i}^{n-1}
$$

Where

$$
\alpha_{i}=s_{i} e^{j \varphi_{i}}
$$


Signal \& Image Processing : An International Journal (SIPIJ) Vol.3, No.6, December 2012

$$
\begin{aligned}
& z_{i}=e^{j \frac{2 \pi}{\lambda} d_{x} \sin \theta_{i} \cos \phi_{i}} \\
& y_{i}=e^{j \frac{2 \pi}{\lambda} d_{y} \sin \theta_{i} \sin \phi_{i}}
\end{aligned}
$$

The azimuth and elevation angles can be obtained uniquely from poles

$$
\left(z_{i}, y_{i}\right), i=1,2, \ldots, N \text {. }
$$

Assume that at random locations a few elements are malfunctioning, and the output is available only from the remaining working elements, neglecting the output of the failed elements. The incomplete and inaccurate data is given as the sampled subset entries, i.e.

$$
\hat{\mathrm{X}}=\mathbf{X}(\Omega)
$$

Where, $\Omega$ is the indexes of the data or the location of the working elements, $\mathbf{X}$ is the Original data and $\hat{\mathrm{X}}$ is the incomplete data corresponding to $\Omega$. The problem is therefore, to estimate the DOAs when the data available is incomplete and inaccurate.

\section{MATRIX COMPLETION}

Recovering a matrix from a sample of its entries is known as Matrix Completion (MC) problem. Suppose, we have a full rank matrix $\mathbf{X}$ of rank $n$, and we have access only to a partial entries of a matrix $\mathbf{X}$ and if the rank of incomplete matrix has a low rank r, then Candes and Rachet in [11], showed that $\mathrm{X}$ can be recovered by using a simple convex optimization [22] problem

$$
\min _{\mathcal{X}_{i, j}=X_{i, j}}\|\mathcal{X}\|_{*} \quad(i, j) \in \Omega
$$

Where, $\Omega$ is the set of locations corresponding to the observed entries, i.e. $(i, j) \in \Omega$ if $\mathrm{X}_{\mathrm{i}, \mathrm{j}}$ is observed.

The functional $\|\mathcal{X}\|_{*}$ is the nuclear norm of the matrix $\mathrm{X}$, which is the sum of its singular values. When the entries are sampled randomly following a uniform distribution, the number of entries $m$ that should be known for perfect recovering the matrix is of the order given by

$$
m \geq C n^{1.2} r \log n
$$

where, $\mathrm{C}$ is some positive numerical constant. The details can be found in [11].

The problem of Matrix Completion is similar to Compressed Sensing (CS) [17] [18]. While MC recovers a rank deficient matrix, CS solves a system of under-determined equations where the 
Signal \& Image Processing : An International Journal (SIPIJ) Vol.3, No.6, December 2012

solution is sparse, i.e. have only a few non-zeros. The CS is also used DOA estimation in [19][20][21] in order to reduce the hardware complexity and the software requirements.

The CS uses $\mathrm{L}_{1}$ minimization on the sparse signal by invoking the Linear Programming to interpolate the signal exactly. In other words, the missing data is interpolated by zeros at respective location. On the other hand, $\mathrm{MC}$ is concerned with the guessing the entries accurately or even exactly that we have not observed [11], which is of our interest in this dissertation.

The Eq. (8) is the tightest convex relation of the NP-hard rank minimization problem

$$
\min _{\mathcal{X}_{i, j}=X_{i, j}} \operatorname{rank}(\mathcal{X}) \quad(i, j) \in \Omega
$$

In [22], the Singular Value Thresholding (SVT) algorithm for approximately solving the nuclear norm minimization problem given by Eq. (8) is developed. The SVT algorithm is as follows.

The problem can be expressed as

$$
\min _{\hat{\mathbf{X}}=P_{\Omega}(\mathrm{X})}\|\mathbf{X}\|_{*}
$$

Where $\|\mathcal{X}\|_{*}$ is called the nuclear norm and is defined as the sum of its singular values, $\mathrm{X}$ is the matrix to be recovered, $\Omega$ is the set of indices of the sampled entries, $\mathrm{P} \Omega$ is a masking operator that selects the entries of $\mathrm{X}$ that are within $\Omega$ and $\hat{\mathrm{X}}$ is the collected partial snapshots.

The inputs to the SVT algorithm are, a parameter $\tau_{\text {svt }}$, step size $\delta$, samples set $\Omega$, sampled entries $\mathrm{X}(\Omega)$ and initializing vector $\mathrm{Y}^{0}=0$. The algorithm is

$$
\begin{aligned}
& \mathbf{X}^{k}=\operatorname{shrink}\left(\mathbf{Y}^{k}, \tau_{s v t}\right) \\
& \mathbf{Y}^{k}=\mathbf{Y}^{k-1}+\delta_{k} \Omega^{T}\left(\hat{\mathbf{X}}-\mathbf{X}^{k}\right)
\end{aligned}
$$

The steps in Eq. (12) are repeated until convergence. shrink $(*)$ is a nonlinear function which applies soft thresholding rule at level $\tau_{\text {svt }}$, to the singular values of the input matrix. The key property here is that for large values of $\tau_{s v t}$, the sequence $\left\{\mathrm{X}^{\mathrm{k}}\right\}$ converges to a solution which very nearly minimizes Eq. (11). Hence, at each step one needs to compute only at most one SVD and perform a few elementary matrix additions.

For shrinkage operator, SVD of Y is taken

$$
\mathbf{Y}=\mathbf{U S V}^{H}, \quad \mathbf{S}=\operatorname{diag}\left(\left\{\sigma_{i}\right\}, 1<i<r\right)
$$

Where $\mathrm{U}$ and $\mathrm{V}$ are the right and the left singular vectors and $\mathrm{S}$ is the singular value matrix. For $\tau_{s v t} \geq 0$, a soft thresholding operator $\mathrm{D}_{\mathrm{T}}$ defined as follows

$$
\begin{aligned}
D_{\tau_{s v t}}(\mathbf{Y}) & =\mathbf{U} D_{\tau_{s v t}}(\mathbf{S}) \mathbf{V}^{H} \\
D_{\tau_{s v t}}(\mathbf{S}) & =\operatorname{diag}\left(\left\{\left(\sigma_{i}-\tau_{s v t}\right)_{+}\right\}, 1<i<r\right)
\end{aligned}
$$


Signal \& Image Processing : An International Journal (SIPIJ) Vol.3, No.6, December 2012

where $(*)_{+}$, is the positive part of $*$. In other words, this operator simply applies the soft thresholding rule to the singular values of Y, effectively shrinking these towards zero. The algorithm is shown in Table 1. The SVT algorithm is listed in Table 1.

\section{2D MATrix EnhanCEMENT MATRIX PENCIL METHOD}

In this section, a brief overview of the 2D matrix enhancement matrix pencil method proposed by Hau in [5] is presented. The method in studied for joint estimation of azimuth and elevation angle estimation.

Table 1 : SVT Algorithm

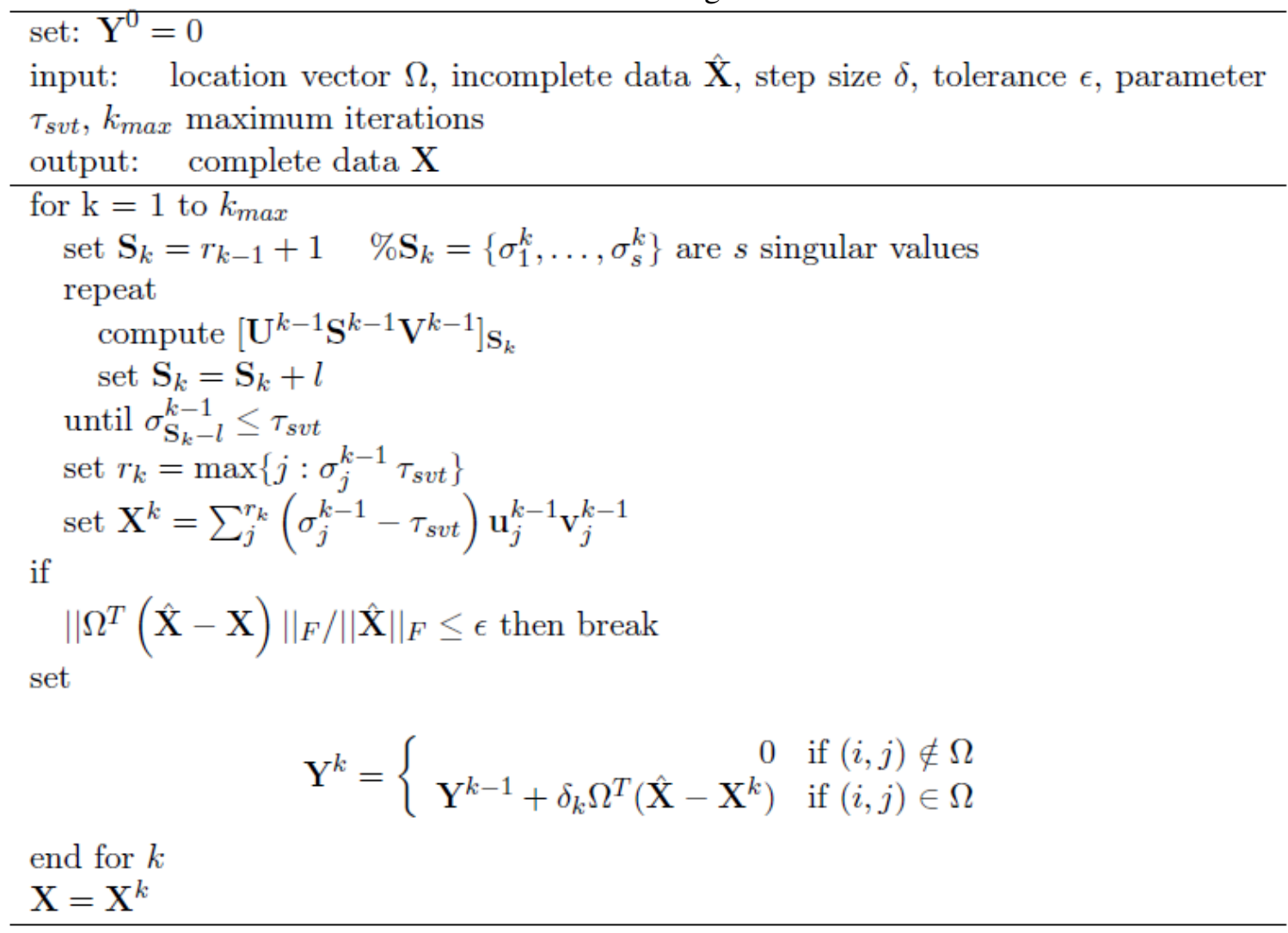

The original data matrix (noiseless) in 3 is defined as follows

$$
\mathrm{X}=\left[\begin{array}{cccc}
x(0 ; 0) & x(0 ; 1) & \ldots & x(0 ; N-1) \\
x(1 ; 0) & x(1 ; 1) & \ldots & x(1 ; N-1) \\
\vdots & \vdots & \ddots & \vdots \\
x(M-1 ; 0) & x(M-1 ; 1) & \ldots & x(M-1 ; N-1)
\end{array}\right]
$$

Using (3) in (15) 
Signal \& Image Processing : An International Journal (SIPIJ) Vol.3, No.6, December 2012

$$
\mathbf{X}=\mathbf{Y A Z}
$$

Where

$$
\begin{gathered}
\mathbf{Y}=\left[\begin{array}{cccc}
1 & 1 & \ldots & 1 \\
y_{1} & y_{2} & \ldots & y_{K} \\
\vdots & \vdots & \ddots & \vdots \\
y_{1}^{M-1} & y_{2}^{M-1} & \ldots & y_{K}^{M-1}
\end{array}\right] \\
\mathbf{A}=\operatorname{diag}\left(\alpha_{1}, \alpha_{2}, \ldots, \alpha_{K}\right) \\
\mathbf{Z}=\left[\begin{array}{cccc}
1 & z_{1} & \ldots & z_{1}^{N-1} \\
1 & z_{2} & \ldots & z_{2}^{N-1} \\
\vdots & \vdots & \ddots & \vdots \\
1 & z_{K} & \ldots & z_{K}^{N-1}
\end{array}\right]
\end{gathered}
$$

The following is noted [5]

- if rank $(\mathrm{X})$ is less than $\mathrm{K},\left\{\mathrm{y}_{\mathrm{i}} ; \mathrm{i}=1, \ldots, \mathrm{K}\right\}$ and $\left\{\mathrm{z}_{\mathrm{i}} ; \mathrm{i}=1, \ldots, \mathrm{K}\right\}$ cannot be both obtained from the principle singular vectors of $X$, and

- the principle singular vectors of $X$ do not contain sufficient information to carry out the pairing between $\mathrm{y}_{\mathrm{i}}$ and $\mathrm{z}_{\mathrm{i}}$.

To overcome the above problems, a enhanced matrix $X_{e}$ is formed from the data matrix $X$ in (15). This process is enhancing the rank condition of the a matrix by a partition-and-stacking process, to form a Hankel block matrix $\mathrm{X}_{\mathrm{e}}$ and is written as

$\mathbf{X}_{e}=\left[\begin{array}{cccc}\mathbf{X}_{0} & \mathbf{X}_{1} & \ldots & \mathbf{X}_{M-L 1} \\ \mathbf{X}_{1} & \mathbf{X}_{2} & \ldots & \mathbf{X}_{M-L 1+1} \\ \vdots & \vdots & \ddots & \vdots \\ \mathbf{X}_{L 1-1} & \mathbf{X}_{L 1} & \ldots & \mathbf{X}_{M-1}\end{array}\right]$

Where 
Signal \& Image Processing : An International Journal (SIPIJ) Vol.3, No.6, December 2012

$$
\mathbf{X}_{m}=\left[\begin{array}{cccc}
x(m ; 0) & x(m ; 1) & \ldots & x(m ; N-L 2) \\
x(m ; 1) & x(m ; 2) & \ldots & x(m ; N-L 2+1) \\
\vdots & \vdots & \ddots & \vdots \\
x(m ; L 2-1) & x(m ; L 2) & \ldots & x(m ; N-1)
\end{array}\right]
$$

Where $\mathrm{X}_{\mathrm{e}}$ is an $\mathrm{L} 1 \times(\mathrm{M}-\mathrm{L} 1+1)$ Hankel block matrix, with

$$
\operatorname{rank}\left(\mathrm{X}_{\mathrm{e}}\right)=\mathrm{K} \geq \operatorname{rank}(\mathrm{X})
$$

And $\mathrm{X}_{\mathrm{m}}$ is an $\mathrm{L} 2 \times(\mathrm{N}-\mathrm{L} 2+1)$ Hankel matrix, and is obtained by windowing the rows of the original data matrix $\mathrm{x}(\mathrm{m} ; \mathrm{n})$. The $\mathrm{L} 1$ and $\mathrm{L} 2$ are the window pencil parameters used to obtain the Hankel matrices of (20) and (21)

The necessary and sufficient condition for rank $\left(X_{e}\right)=K$ is

$$
\begin{array}{r}
\mathrm{L} 1 \mathrm{~L} 2 \geq \mathrm{K} \\
(\mathrm{M}-\mathrm{L} 1+1)(\mathrm{N}-\mathrm{L} 2+1) \geq \mathrm{K}
\end{array}
$$

assuming that $\mathrm{K}$ is known

To extract the 2D poles the Singular Value Decomposition (SVD) is applied to Xe to obtain

$$
\begin{aligned}
\mathbf{X}_{e} & =\sum_{i=1}^{\min } \sigma_{i} \mathbf{u}_{i} \mathbf{v}_{i}^{H} \\
& =\mathbf{U}_{s} \Sigma_{s} \mathbf{V}_{s}^{H}+\mathbf{U}_{n} \Sigma_{n} \mathbf{V}_{n}^{H}
\end{aligned}
$$

where $\min =\min \left(\mathrm{L} 1 \mathrm{~L} 2,((\mathrm{M}-\mathrm{L} 1+1)(\mathrm{N}-\mathrm{L} 2+1))\right.$ which is the smaller dimension of $\mathrm{X}_{\mathrm{e}}, \mathrm{U}_{\mathrm{s}}$, $\sum_{\mathrm{s}}$, and $\mathrm{V}_{\mathrm{s}}$ contains the $\mathrm{K}$ principal components and $\mathrm{U}_{\mathrm{n}}, \sum_{\mathrm{n}}$ and $\mathrm{V}_{\mathrm{n}}$ contains the remaining nonprincipal components.

If $\operatorname{rank}\left(\mathrm{X}_{\mathrm{e}}=\mathrm{K}\right)$

$$
\operatorname{range}\left(\mathbf{X}_{e}\right)=\operatorname{range}\left(\mathbf{U}_{s}\right)
$$

and

$$
\operatorname{range}\left(\mathbf{X}_{e}^{H}\right)=\operatorname{range}\left(\mathbf{V}_{s}^{H}\right)
$$

The above properties are used to extract the poles.

Further, to extract $y_{i}^{\prime} s$, we define

$$
\begin{aligned}
& \mathbf{U}_{1}=\mathbf{U}_{s}(1: L 2-1) \\
& \mathbf{U}_{2}=\mathbf{U}_{s}(2: L 2)
\end{aligned}
$$


Signal \& Image Processing : An International Journal (SIPIJ) Vol.3, No.6, December 2012

If the above condition in (22) of the pencil parameters are satisfied, we can now formulate as a Generalized Eigenvalue Problem (GEP)

$$
\mathbf{U}_{2}-\lambda \mathbf{U}_{1}
$$

Where $\lambda$ are the generalized eigenvalues of the corresponding matrix pencil, i.e. $\lambda=y_{\mathrm{i}}$, $\mathrm{i}=1,2, \ldots, \mathrm{k}$

Next, to extract to extract the $x_{i}^{\prime} s$ we define

$$
\mathbf{U}_{s P}=\mathbf{P} \mathbf{U}_{s}
$$

Where $\mathrm{P}$ is the permutation matrix. And

$$
\begin{aligned}
\mathbf{U}_{1 P} & =\mathbf{U}_{s P}(1: L 1-1) \\
\mathbf{U}_{2 P} & =\mathbf{U}_{s P}(2: L 1)
\end{aligned}
$$

Then the matrix pencil is

$$
\mathbf{U}_{2 P}-\lambda \mathbf{U}_{1 P}
$$

where $\lambda$ are the generalized eigenvalues of the corresponding matrix pencil, i.e. $\lambda=x i, i=1,2, \ldots$ ., $\mathrm{K}$

The azimuth and elevation angles are obtained from the poles $\left(\mathrm{y}_{\mathrm{i}}, \mathrm{z}_{\mathrm{i}}\right)$ obtained after pairing [5]. These angles are obtained from

$$
\begin{aligned}
G_{i} & =\operatorname{angle}\left(x_{i}\right) / \pi \\
E_{i} & =\operatorname{angle}\left(y_{i}\right) / \pi \\
\theta_{i} & =\operatorname{atan}\left(E_{i} / G_{i}\right) \\
\phi_{i} & \left.=\operatorname{asin}\left(\sqrt{(} E_{i}^{2}+G_{i}^{2}\right)\right)
\end{aligned}
$$

\section{2D MATRIX COMPleted MATRIX PENCIL METHOD}

We can now extend the 2D MP method for the faulty UPA. Due to wear and tear, a few elements at random locations have failed to produce the output. The resulting output of a UPA is a incomplete data, and is given as

$\hat{X}_{i, j}=X_{i, j} ; \quad i, j \in \Omega \quad$ if $X_{i, j}$ is observed

where, $\Omega$ is the set of locations corresponding to the observed data output, from the functioning elements. The conventional 2D MP method described in the previous section cannot be applied directly on such an incomplete data. As the method relies completely on the completeness of the data, the extension of the conventional MP method for a faulty array is achieved by perfectly recovering the missing data to complete the data matrix, which is further processed to estimate the DOAs. 
Signal \& Image Processing : An International Journal (SIPIJ) Vol.3, No.6, December 2012

The problem of recovering the complete data matrix can be written as

$$
\min _{\hat{\mathbf{x}}=\mathbf{x}(\Omega)}\|\mathbf{X}\|_{*}
$$

$\hat{\mathrm{X}}$ is the data collected from working elements, $\Omega$ is the location set whose entries are the location of the elements that are working in a UPA, $\mathrm{X}$ is the matrix to be recovered and the operator $\mathrm{X}(\Omega)$ represents the data collected from only the working elements. The solution to the problem in Eq. (33) is obtained using Singular Value Thresholding (SVT) algorithm proposed by Cai, Candes and Shen [22].

\subsection{Summary of the proposed MCMP method}

The algorithms described in the previous section assume noiseless data. In this section, we summarize the MCMP algorithm for the noisy data. For convenience, we use the notation $\mathrm{X}$ for the data (noisy). The studied MCMP algorithm is summarized in Table. 2

Table 2: Summary of the MCMP method

\begin{tabular}{|c|c|}
\hline Algorithm & Complexity \\
\hline $\begin{array}{l}\text { Construct the complete data matrix } \mathrm{X} \text { from } \\
\text { the incomplete data matrix } \mathrm{X} \text {, applying the } \\
\text { SVT algorithm } \\
\text { Construct the enhance matrix } \mathrm{X}_{e} \text { using } \\
\text { Eq. (20) }\end{array}$ & $4 M y M z^{2}+8 M z^{3}$ \\
\hline $\begin{array}{l}\text { Compute } \mathrm{SVD} \text { of } \mathrm{X}_{e} \text { to obtain } \mathbf{U}_{s}, \Sigma_{s} \text {, and } \\
\mathbf{V}_{s}, N \text { principal singular components } \\
\text { Compute Eq. (26) and solve Eq. }(27) \text { to obtain } \\
y_{j}^{\prime} s\end{array}$ & $\begin{array}{l}4 L 1^{2}(M y-L 1+1)^{2}+8 L 1(M y-L 1+ \\
1)^{2}+9(M y-L 1+1) \\
(L 1+1)^{2}\end{array}$ \\
\hline $\begin{array}{l}\text { Compute Eq. (29) and solve Eq. (30) to obtain } \\
z_{i}^{\prime} s\end{array}$ & $(L 1+1)^{2}$ \\
\hline $\begin{array}{l}\text { Pair the poles } \\
\text { Calculate the } \theta_{i} \text { and } \phi_{i} \text { using Eq. (31) } \\
\text { Check for Convergence } \\
\text { end }\end{array}$ & $N^{3} L 1 L 2$ \\
\hline $\begin{array}{l}\text { Total }=4 M N^{2}+8 M z^{3}+4 L 1^{2}(M y-L 1+ \\
1)+(L 1+1)^{2}+N^{3} L 1 L 2\end{array}$ & \\
\hline
\end{tabular}

\section{SIMULATION RESULTS}

In this section, computer simulations are provided to illustrate the performance of the proposed technique. Two complex exponential signals of equal magnitude and phase corrupted by additive white Gaussian noise (AWGN) are considered. The signals are impinging on the array from azimuth and elevation angles of $\left[10^{\circ}, 25^{\circ}\right]$ and $\left[30^{\circ}, 40^{\circ}\right]$ respectively. The number of elements are $\mathrm{My}=\mathrm{Mz}=20$ and the distance between the elements on both the axis $\mathrm{d}_{\mathrm{x}}=\mathrm{d}_{\mathrm{y}}=\lambda / 2$. The results are based on 100 trails. We assume that a few number of elements at random locations have failed to produce to the output. There are techniques available for knowing the number of elements failed and their locations and can be found in [23]. Three examples are considered to evaluate the performance. 
Signal \& Image Processing : An International Journal (SIPIJ) Vol.3, No.6, December 2012

In the first example, the performance of the proposed 2D MCMP DOA estimation method is evaluated for various number of working element. Three cases of number of working elements is assumed. Unlike, the examples in the previous chapters, we in this chapter, assume more number of element failures, i.e., reduced number of working elements at random locations, to know the effectiveness of the proposed 2D MCMP DOA estimation algorithm. The number of elements feeding the output, i.e., the number of working elements considered are 240 elements out of 400 elements $(20 \times 20$ UPA), 200 elements out of 400 elements and 160 elements out of 400 elements. In other words, we have only $60 \%, 50 \%$ and $40 \%$ of observations. The signals are corrupted due to AWGN of $20 \mathrm{~dB}$. The estimated azimuth and elevation angles are plotted on a scatter plot shown in Fig. 2 for all the three cases respectively.

It can be observed that, for $60 \%$ and $50 \%$ observations the method is successful in estimating the angles. Whereas, for $40 \%$ observations, the method shows less accuracy. Therefore, for the assumed signal environment, the proposed method estimates the angles with at least $50 \%$ of observation or using 200 functioning elements or in other words the algorithm effectively handles up to 200 malfunctioning elements. Provided that the location of the working elements is known.

The performance of the proposed algorithm is further evaluated using the RMSE vs SNR plot for the estimated azimuth and elevation angles. The SNRs considered are $5 \mathrm{~dB}, 10 \mathrm{~dB}, 15 \mathrm{~dB}, 20 \mathrm{~dB}$ and $25 \mathrm{~dB}$. The number of working elements considered are 400 elements (all elements are functioning), 360 elements, 280 elements and 200 elements are considered working. From Fig. 3 it can be seen that for all the cases of observations the performance is almost similar. The proposed is able to handle a reasonable number of element failures. Furthermore, the performance is at its best under high SNR.

In the final example, we considered the RMSE plot between the estimated angles and percentage of the working elements. The SNRs assumed are $30 \mathrm{~dB}, 20 \mathrm{~dB}$ and $10 \mathrm{~dB}$ and the number of working elements assumed are $[120,160,200,240,280,320,360]$ elements at random locations. Fig. 4 shows the RMSE plot for elevation and azimuth angles. We observe that the proposed algorithm is able to estimate the angles at low SNRs and a reasonable size of working elements. It can be seen that, for 120 functioning elements at random locations, the algorithm is moderately accurate at low SNR values, however, at high SNR values the accuracy is improved. Therefore, from all the three examples, it can be observed if the noise effect is reduced considerably, the performance is better.

\section{CONCLUSION}

A Matrix Completion Matrix Pencil method for the two dimensional DOA estimation has been developed for the extending the conventional two dimensional Matrix Pencil method, to handle the element failure in the UPA. The Matrix Completion procedure is used to impute the missing data to reconstruct the complete data and later estimate the DOAs. The computational complexity when compared with the conventional 2D matrix pencil method is slightly increased. The increase is in computing the singular vectors for Matrix completion procedure. The proposed algorithm is able to estimate the DOAs when only a fraction of the elements are working. The algorithm shows improved performance when the SNR is very low. 
Signal \& Image Processing : An International Journal (SIPIJ) Vol.3, No.6, December 2012

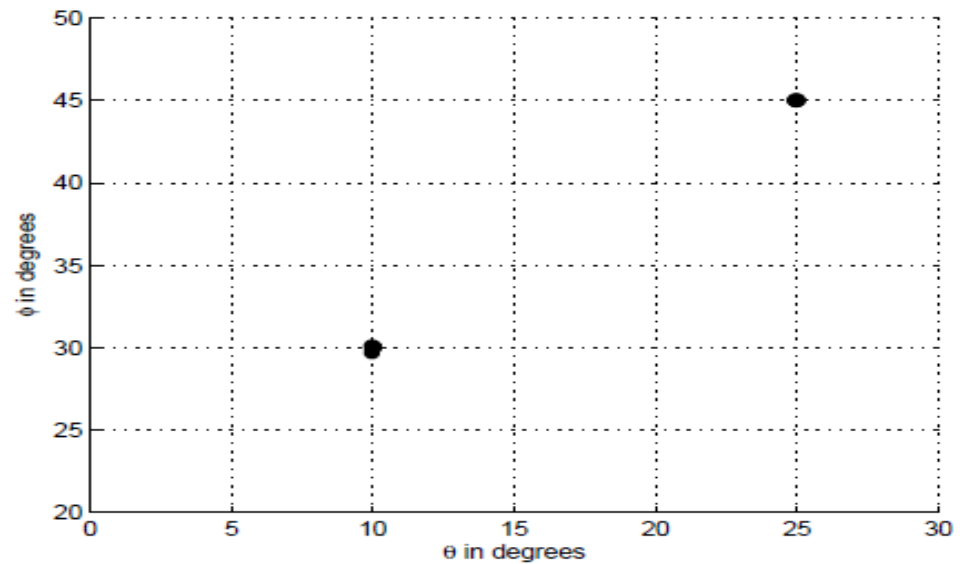

(a) 240 elements are working

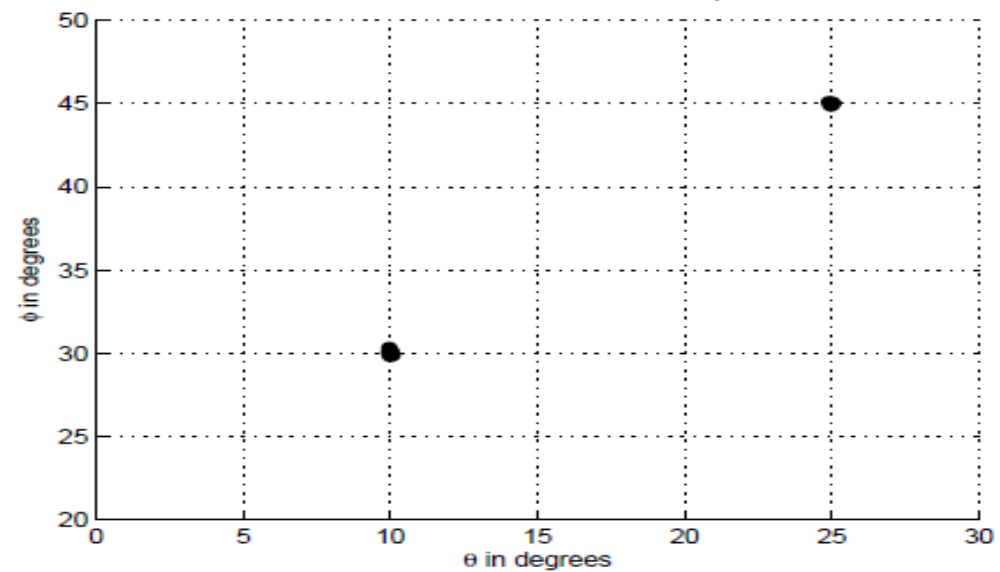

(b) 200 elements are working

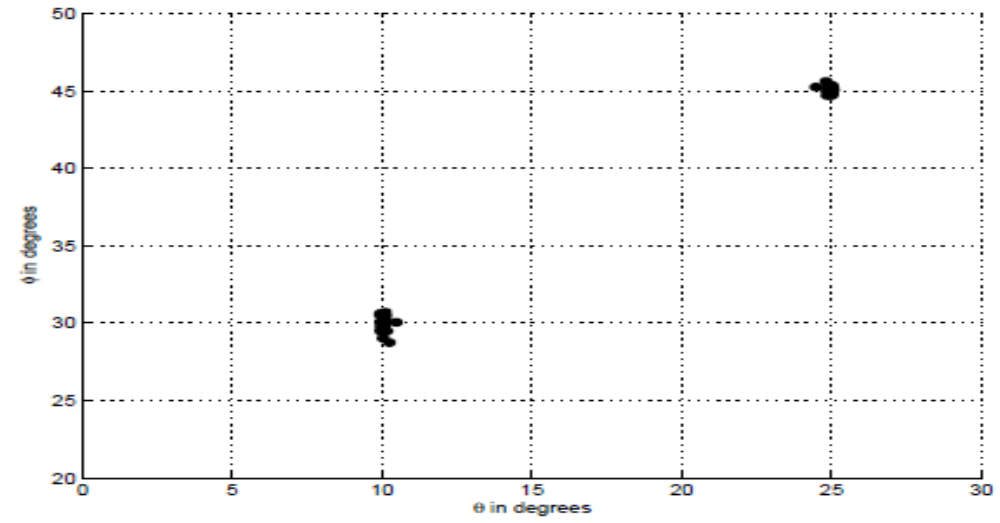

(c) 160 elements are working

Figure 2: Scatter plot of the estimated azimuth and elevation angles. Two signals are impinging from $10^{\circ}$ and $25^{\circ}$ on the array of size $20 \times 20$ elements, SNR is $20 \mathrm{~dB}$. 
Signal \& Image Processing : An International Journal (SIPIJ) Vol.3, No.6, December 2012

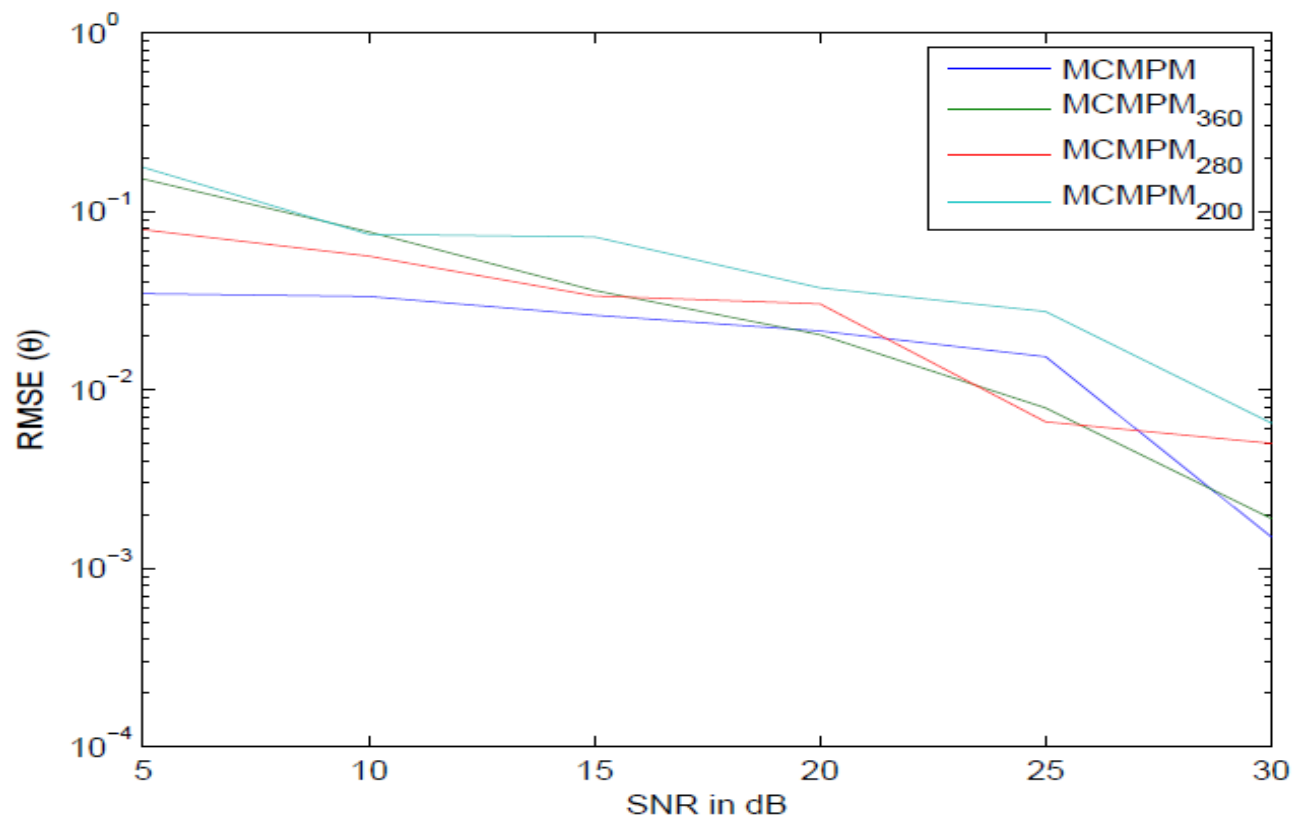

(a) Azimuth angles

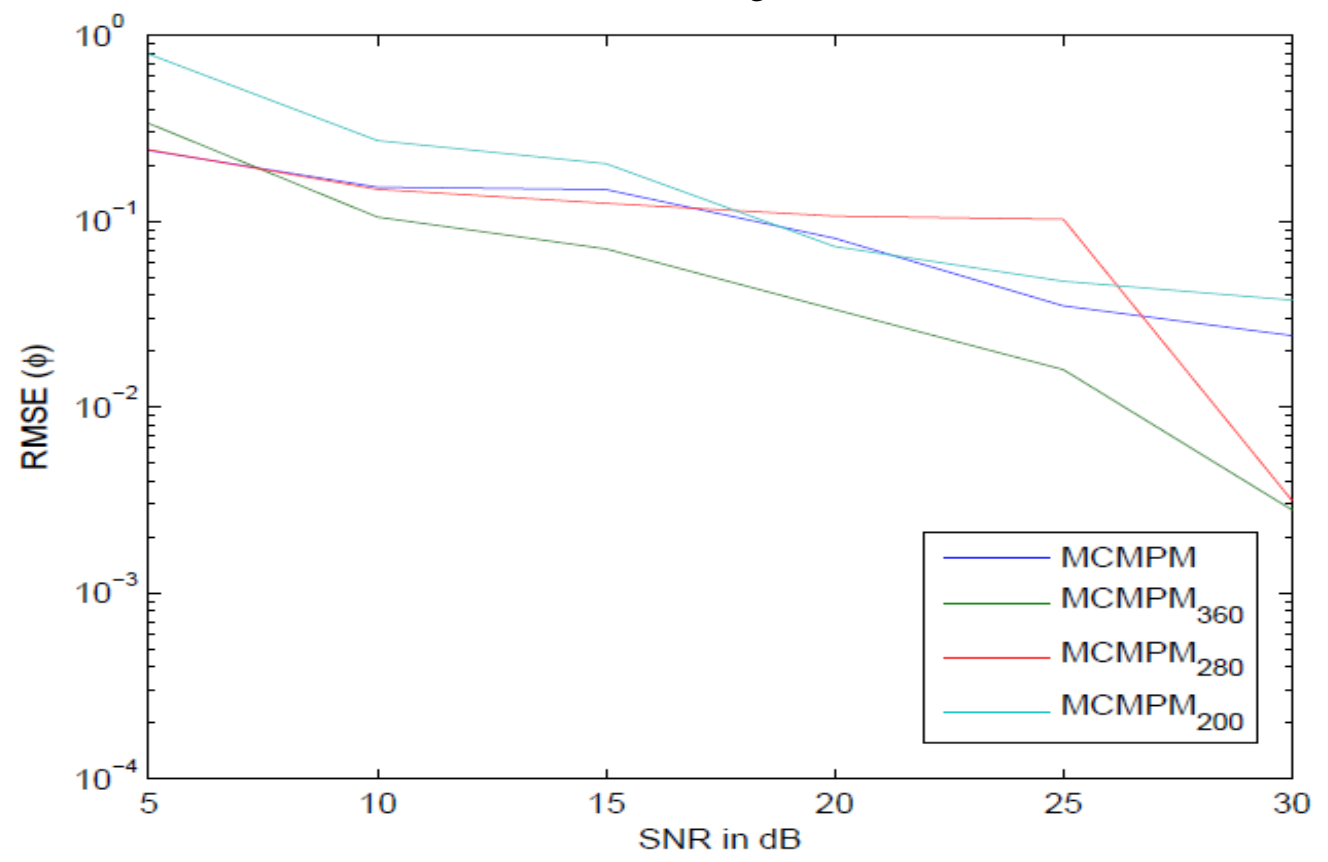

(b) Elevation angles

Figure 3: RMSE vs SNR plot for various working elements. 
Signal \& Image Processing : An International Journal (SIPIJ) Vol.3, No.6, December 2012

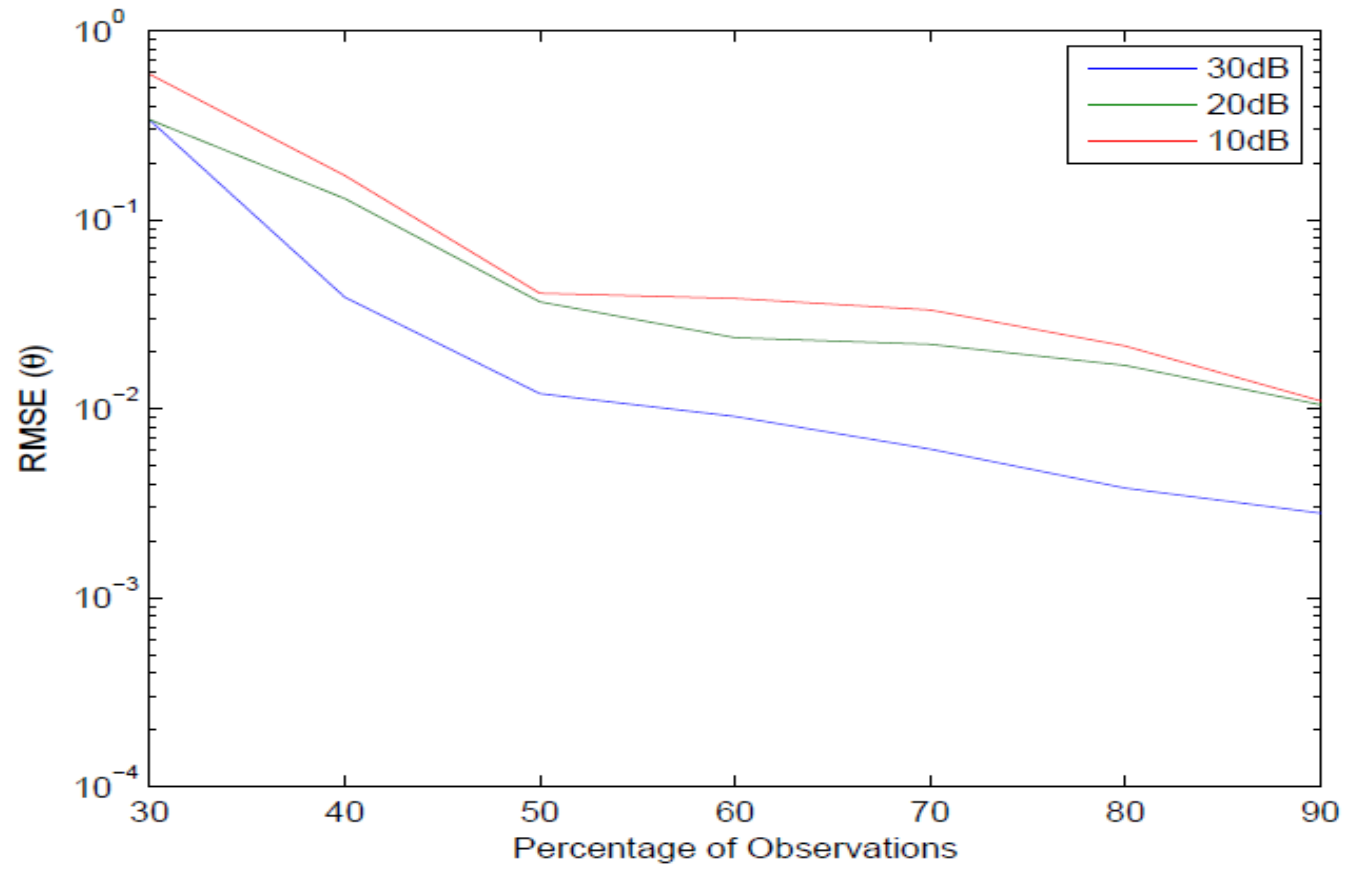

(a) Azimuth angles

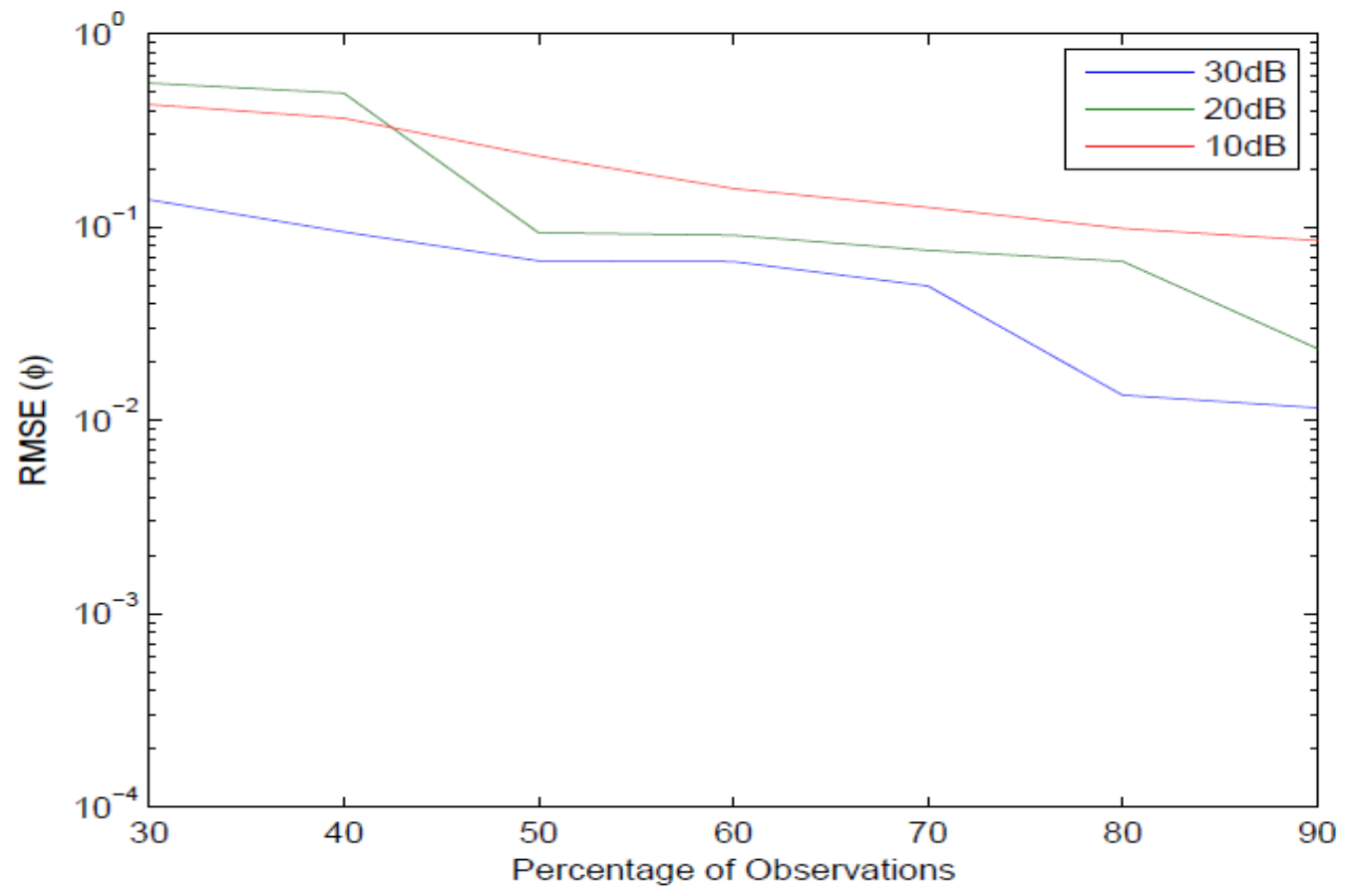

(b) Elevation angles

Figure 4: RMSE vs SNR plot for various working elements. 
Signal \& Image Processing : An International Journal (SIPIJ) Vol.3, No.6, December 2012

\section{REFERENCES}

[1] Yerriswamy T. and S. N. Jagadeesha. Fault tolerant matrix pencil method for direction of arrival estimation. Signal and Image Processing : An International Journal, 2(3):42-46, 2011.

[2] Q. Cheng and Y. Hua. Further study of the pencil-music algorithm. IEEE Trans. Aerospace Electronic Systems, 32(1):284-299, 1996.

[3] V. S. Kedia and B. Chandna. A new algorithm for 2-d doa estimation. Signal Processing, 60(3):325$332,1997$.

[4] Junli Liang and Ding Liu. Joint elevation and azimuth direction finding using l-shaped array. IEEE Trans. on Antennas and Propagation, 58(6):2136-2141, 2010.

[5] Y. Hua. Estimating two dimensional frequencies by matrix enhancement and matrix pencil. IEEE Trans. Signal Processing, 40(9):2267-2280, 1992.

[6] N. Yilmazer and T. K. Sarkar. 2-d unitary matrix pencil method for efficient direction of arrival estimation. Digital signal processing, 16:767-781, 2006.

[7] E. G. Larsson and P. Stoica. High-resolution direction finding: The missing data case. IEEE Trans. Signal Processing, 49(5):950-958, 2001.

[8] N. Sundararajan, S. Vigneshwaran, and P. Saratchandran. Direction of arrival (doa) estimation under array sensor failure using a minimal resource allocation neural network. IEEE Tran. on Antennas and Propagation, 55(2):334-343, 2007.

[9] B. K. Yeo and Y. Lu. Array failure correction with a genetic algorithm. IEEE Trans. Antennas and Wave Propagation, 47(5):823-828, 1999.

[10] T. W. Pirinen, J. Yli-Hietanen, P. Pertila, and A. Visa. Detection and compensation of sensor malfunction in time delay based direction of arrival estimation. In Proc. Int. Symp. on Circuits and Systems, ISCAS 04, pages IV-872-875, 2004.

[11] E. J. Candes and B. Recht. Exact matrix completion via convex optimization. Foundational computational Mathematics, 9(6):717-772, 2008.

[12] Stephen Boyd and Lieven Vandenberghe. Convex Optimization. Cambridge University Press, 2008.

[13] J.Matingley and S. Boyd. Real time convex optimization in signal processing : Recent advances that make it easier to design and implement algorithms. IEEE Signal Processing Magazine, pages 50-61, 2009.

[14] B. Savas and D. Lindgren. Rank reduction and volume minimization approach to state-space subspace system identification. Signal Processing, 86(11):3275-3285, 2006.

[15] A. Gilbert, S. Muthukrishnan, and M. Strauss. Improved time bounds for near-optimal sparse fourier representation. In Wavelets XI at SPIE Optics and Photonics, San Diego, California, 2005.

[16] Ziqian Dong, Santhanakrishnan Anand, and Rajarathnam Chandramouli. Estimation of missing rtts in computer networks: Matrix completion vs compressed sensing. Computer Networks, 5:3364-3375, 2011.

[17] E. J. Candes and M. B. Wakin. A introduction to compressive sampling. IEEE Signal Processing Magazine, 25(2):21-30, 2008.

[18] D. L. Donoho. Compressed sensing. IEEE Trans. on Information Theory, 52(4):1289-1306, 2006.

[19] Ying Wang, Geert Leus, and Ashish Pandharipande. Direction estimation using compressive sampling array processing. In Proc. of IEEE/SSP 15th Workshop on Statistical Signal Processing, pages 626-629, 2009.

[20] A. C. Gurbuz, J. H. McClellan, and V. Cevher. A compressive beamforming method. In Proc. of IEEE International Conference on Acoustics, Speech and Signal Processing, pages 2617-2620, 2008.

[21] V. Cevher, A. Gurbuz, J. McClellan, and R. Chellappa. Compressive wireless array for bearing estimation. In Proc. of IEEE International Conference on Acoustics, Speech and Signal Processing, pages 2497-2500, 2008.

[22] E. J. Candes, J. F. Cai, and Z. Shen. A singular value thresholding algorithm for matrix completion. SIAM Journal of Optimization, 20(4):1956-1982, 2008.

[23] R. Iglesias and et al. Element failure detection in linear antenna arrays using case based reasoning. IEEE Antennas and Propagation Magazine, 50(4):198-204, 2008. 
Signal \& Image Processing : An International Journal (SIPIJ) Vol.3, No.6, December 2012

\section{Authors}

Yerriswamy T. received B.E. in Electronics and Commn. Engg. from Gulbarga University, Karnataka, India in 2000. He received his M.Tech. in Network and Internet Engg. from Visvesvaraya Technological University, India in 2006. He is currently working towards PhD from Visvesvaraya Technological University, India. At present he is Asst. Prof. at Proudhadevaraya Institute of Technology (affiliated to Visvesvaraya Technological University), Hosapete, Karnataka, India.

S.N. Jagadeesha received his B.E. in Electronics and Commn. Engg. from B.D.T College of Engg., Davanagere affiliated to Mysore University, Karnataka, India in 1979, M.E. from Indian Institute of Science, Bangalore specializing in Electrical Science, India in 1987 and $\mathrm{PhD}$ in Electronics and Computer Engg. from University of Roorkee, Roorkee, India in 1996. He is an IEEE member. His research interest includes Array Signal Processing, Wireless Sensor Networks and Mobile Communications. He has published and presented many papers on Adaptive Array Signal Processing and Direction-of-Arrival estimation. Currently he is professor in the department of Computer Science and Engg, Jawaharlal Nehru National College of Engg. (affiliated to Visvesvaraya Technological University), Shivamogga,Karnataka, India. 